\title{
Quantifying the effect of mixing on the mean age of air in CCMVal-2 and CCMI-1 models
}

Simone Dietmüller et al.

Correspondence to: Simone Dietmüller (simone.dietmueller@dlr.de)

The copyright of individual parts of the supplement might differ from the CC BY 4.0 License. 


\section{Inconsistencies in the calculation of residual vertical velocities in CCM data}

The model inter-comparison projects CCMVal-2 and CCMI-1 include data requests for the residual velocities $\bar{v}^{*}$ and $\overline{\mathrm{w}}^{*}$. The $\overline{\mathrm{w}}^{*}$ fields are specified to be given in units of $m \mathrm{~s}^{-1}$. However, as most models use hybrid-pressure coordinates, the vertical velocity $\bar{\omega}^{*}$ in $\mathrm{Pa} \mathrm{s} \mathrm{s}^{-1}$ is usually available and has to be transformed to $\mathrm{m} \mathrm{s}^{-1}$. The exact transformation of $\omega$ to $\mathrm{w}$ is given by:

$5 \quad \omega \approx \frac{d p}{d t}=\frac{\partial z}{\partial t} \frac{\partial p}{\partial z}=\mathrm{w} \frac{-p g}{R T}$

I.e. on a given pressure level $p$ the transformation is dependent on the local temperature $T$. Alternatively, a constant scale height assumption can be made for the transformation:

$\omega \approx \mathrm{w} \frac{-p}{H}$

In the CCMI data request a scale height of $\mathrm{H}=6950 \mathrm{~m}$ was suggested. Fig. S1 compares the profile of tropical mean $\mathrm{w}^{*}$ from one model (EMAC) calculated with the actual density (Equ. 1, red line) and with the scale height (Equ. 2, black line). Differences in the lower stratosphere (between 100 to $70 \mathrm{hPa}$ ) are more than $20 \%$. Upon close inspection of delivered CCMI model output, it turned out that some model data was processed converting vertical velocities with the actual density while others used a fixed scale height. Given the large deviations of the two methods, this inconsistency has to be taken into account for a quantitative model comparison. In the following we give an outline how those inconsistencies can be detected, and how a consistent model comparison can be achieved.

Given continuity, the residual vertical velocity is linked to the meridional velocity:

$$
\frac{\partial \bar{v}^{*}}{\partial y}+\frac{\partial \bar{\omega}^{*}}{\partial p}=0
$$

Therefore, it is possible to deduce the residual vertical velocity $\bar{\omega}^{*}$ from the residual meridional velocity by vertical integration and meridional derivation. This vertical velocity $\bar{\omega}^{*}$ can then again be converted to $\bar{w}^{*}$ using the given scale height. By comparing the given $\overline{\mathrm{w}}^{*}$ from the model output to the $\overline{\mathrm{w}}^{*}$ that is calculated from $\bar{v}^{*}$ (referred to as $\overline{\mathrm{w}}^{*}{\overline{v^{*}}}^{-}$in the following) it can be tested whether the same scale height was used originally in the calculation of $\bar{w}^{*}$. As shown in Fig. S2, tropical mean $\bar{w}^{*}$ at $70 \mathrm{hPa}$ as delivered for the CCMI models agrees with the derived $\overline{\mathrm{w}}^{*}{ }_{v^{*}}$ within $<10 \%$ for 4 models, while in 3 models to difference is close to or above $20 \%$. After consulting the modeling groups it turned out that the three models with the largest difference (EMAC, NIWA-UKCA, SOCOL) indeed used the actual density to calculate $\bar{w}^{*}$ (i.e. Equ. 1) instead of 

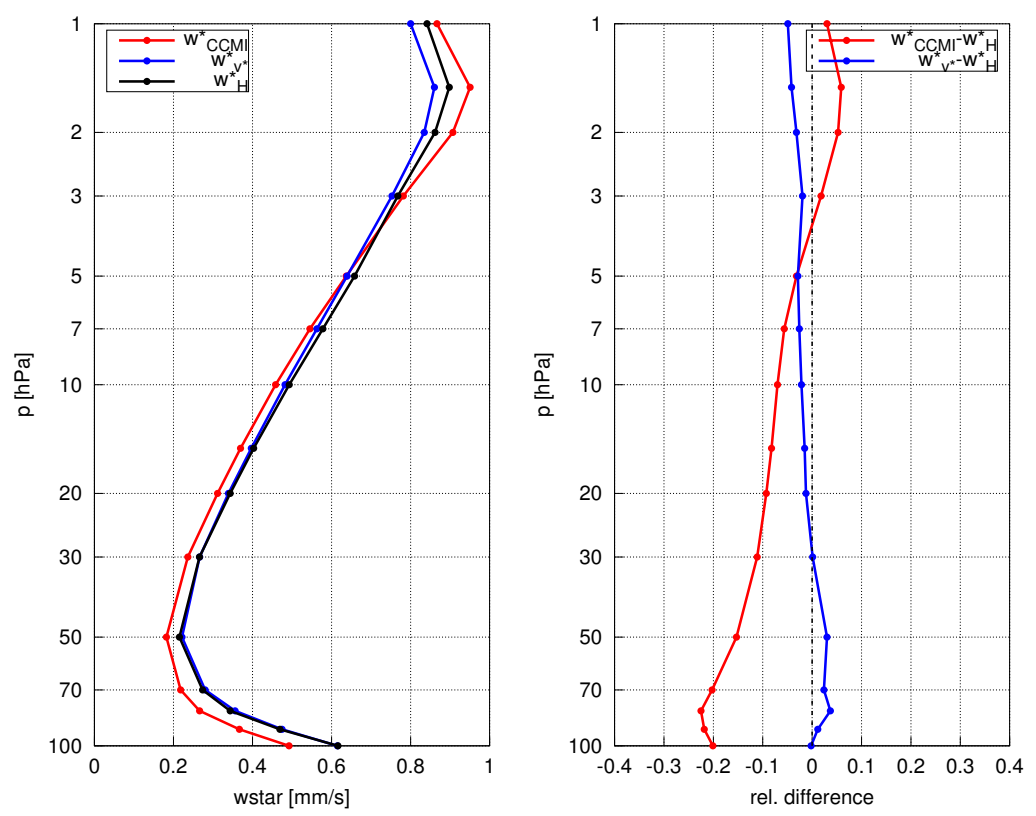

Figure S1. Profile of climatological mean $\overline{\mathrm{w}}^{*}$ over $20^{\circ} \mathrm{S}-20^{\circ} \mathrm{N}$ for EMAC-L90. Left: $\overline{\mathrm{w}}^{*}$ CCM I delivered to CCMI with actual density used in the transformation (red), $\overline{\mathrm{w}}^{*}{ }_{H}$ with scale height used for the transformation (black) and $\overline{\mathrm{w}}^{*} \overline{v *}^{*}$ derived from $\overline{v *}$ (blue). Right: relative difference between $\overline{\mathrm{w}}^{*}{ }_{C C M I}$ to $\overline{\mathrm{w}}^{*}{ }_{H}$ (red) and relative difference between $\overline{\mathrm{w}}^{*} \bar{v}^{*}$ and $\overline{\mathrm{w}}^{*}{ }_{H}$ (blue).

the fixed scale height. This inconsistent calculation method inhibits quantitative model comparison of the delivered $\mathrm{w}^{*}$ fields, as differences due to different methods are of similar magnitude than the model differences (as can be seen from Fig. S2).

To ensure consistent treatment of model data, we used in our study the $\overline{\mathrm{w}}^{*}$ fields derived from $\overline{v^{*}}$. While this calculation also introduces errors due to the vertical integration and meridional derivation, it is ensured that the model data is treated in the same way for all models. The error made in the derivation of ${\overline{w^{*}}}^{*}$ is shown for the EMAC model in the right panel of Fig. S1: the difference between the $\overline{\mathrm{w}}^{*}$ calculated directly from $\overline{\omega *}$ with the scale height and the $\overline{\mathrm{w}}^{*} \bar{v}^{*}$ derived from $\bar{v}^{*}$ (blue line) is smaller $5 \%$, i.e. much smaller than the difference due to scale height versus density transformation.

Based on those results we strongly encourage anyone working with residual velocities from multi-model comparison projects to:

1. Check if the given residual vertical velocities $\overline{\mathrm{w}}^{*}$ are consistently calculated by comparison to the derived $\overline{\mathrm{w}}^{*}{ }_{v *}$ from $\bar{v}^{*}$

2. If necessary, use those derived $\overline{\mathrm{w}}^{*}{ }_{v *}$ values for quantitative model comparison 


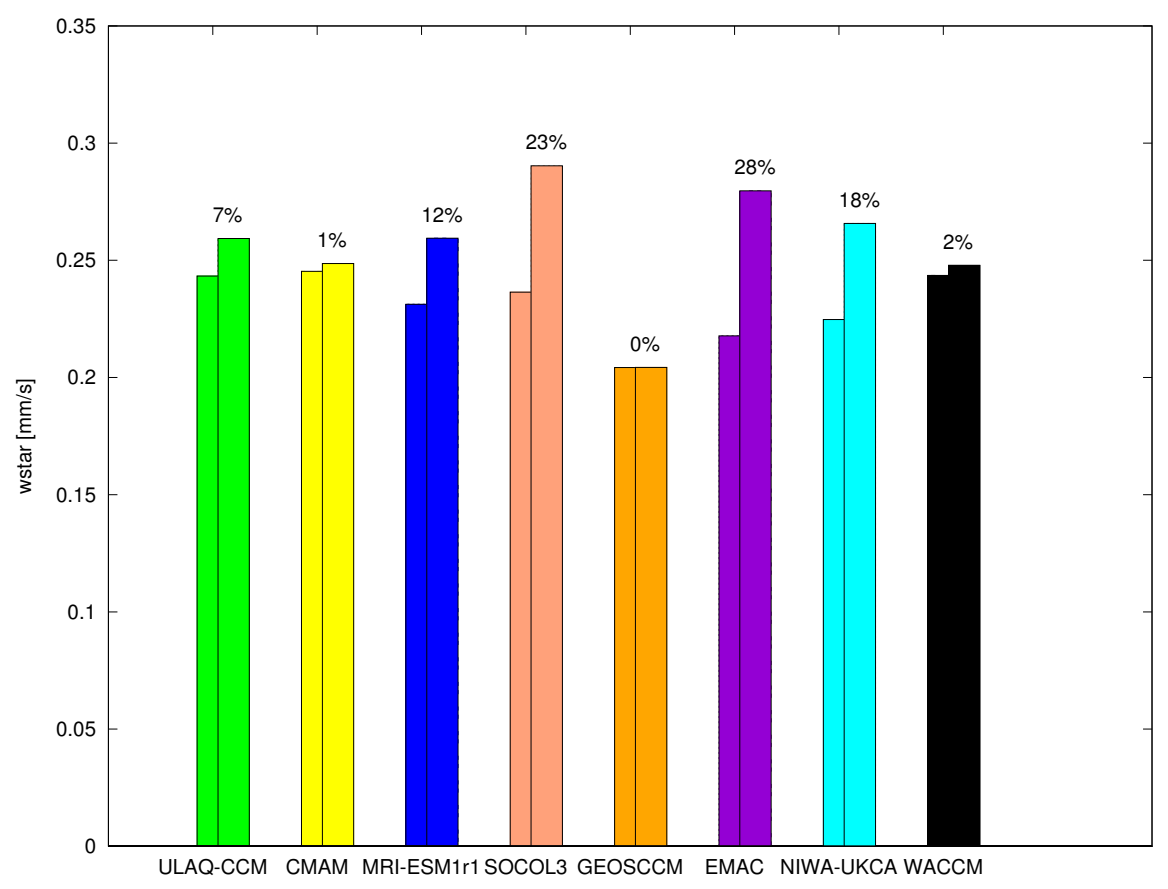

Figure S2. Climatological mean $\overline{\mathrm{w}}^{*}$ over $20^{\circ} \mathrm{N}-20^{\circ} \mathrm{S}$ at $70 \mathrm{hPa}$ for all CCMI REF-C1 model simulations used in this study. The left bar is the $\overline{\mathrm{w}}^{*}$ delivered by the models, the right bar is $\overline{\mathrm{w}}^{*} \bar{v}^{*}$ derived from $\bar{v}^{*}$. The number at the top is the percentage difference. 